\title{
Applied Study on the Reproduction of Visual Perception in Photographic Creation
}

\author{
Zhenya Zhang \\ Zhongyuan University of Technology \\ Zhengzhou, China
}

\begin{abstract}
This article investigates visual concepts related to an indiviual's eye, brain and perception of the world in relation to the visual stimulation of photographic images. The artist knows how to study, view, and to create the image is being previewed by an audience. When an artist creates a new image structure, this new visual image will influence and change the way one may view the objective world.
\end{abstract}

Keywords-visual perception; photography; gestalt; conceptual photography

\section{The BACKGRound, Purpose AND SignificANCE TO THE STUDYING OF VISUAL PERCEPTION APPLIED IN PHOTOGRAPHIC CREATION}

Human culture can be divided into two types from the point of visual perception: characters and images. A character is a symbol system that can be used for communicating thoughts and awareness, and it is studied in literature and art, philosophy, language. An image is studied in visual perception, which is used by the human before the characters appearing in ancient times.

An image is a language tool contains valuable information, and it can be interpreted and applied to the visual arts. The three abilities of image are: observation, interpreting visual images and image expression. These are inseparable abilities. One who lacks sharp observation, the ability of interpreting images, and applying visual images is less likely to be good at painting, sculpture, design, photography, cinematography, and so on. Therefore, the visual perception is necessary to study in order to interpret human visual experiences, revealing how humans view the world and the living environment around them. By studying, ways and habits of viewing the world may change towards a positive direction.

By combining studying how photography displays the world and how people observe said photography with theoretical research on visual perception, we've discovered that photography does not display objective reality. Since the camera cannot be objective, the photography art is also not objective.

Much has been discovered amongst all aspects of visual perception from the numerous research papers that have been published. Some psychological textbooks summarize the research results, but most of the research materials are rarely linked to the artistic and technical aspects of photography.
By means of common recognition and comparative studying between photography mode and visual perception mechanism, we put forward some new ideas and methods of photography creation in order to provide basis and methods for photography creation.

\section{THE APPLICATION AND PERFORMANCE OF VISUAL PERCEPTION IN THE CREATION OF PHOTOGRAPHY}

Visual communication requires an eye, as well the mind which make sense of the received information. Flexible, curious minds can remember and use visual information creatively and understand the world and its visual representations. This will help one analyze the various images and take outstanding photographs.

The eyes are the basis to understanding the physical world. Although they can see an image physiologically, it does not mean that there is interest in looking at the image. The sight lack of feeling is not a true visual image. Therefore, the vision should show implied parts of the image, rather than just surface parts. Excellent photographs can express inherent meaning using vision; the viewer will achieve an abstract understanding which can transform the image into symbolization, namely visual language.

Like sensation and perception, thinking is also a response to objective reality. However, sensation and perception is a direct response to the objective reality with external characteristics of objective reality and external links, while thinking is indirect and general response which is common and essential characteristics and inner links to the objective reality.

Photographers often take attention on visual thinking. As early as 1929, Edward Weston has told us that the texture, movement, light, proportion of every detail of photos should be completely imagined in advance prior to exposure, which was same with the concept form Ansel Adams and Minou Art originates in a particular way of thinking. A human being's response to art has already been instinctive and purposeful. Therefore, the photography creation should make people clearly understand the art's expression. It shouldn't make people feeling obscure and deviated.

The image from a camera is the result of mechanical recording, and people can analyze the content of the image by abstract thinking, by this time they can recognize the image. If an image is absence of abstract thinking, then it 
will quickly disappear from the mind. Therefore, when only one image reaches the retina level of understanding, it is not enough. The lack of images to form an abstract picture can have negative effects. If an image couldn't appear dreams and other imagery, then, it may not be a quality image. An image without sensation imagery is not a complete picture.

In 1962, an experiment had been conducted with a cat brain by David Hubel and Torsten Wieser at John Hopkins University in Baltimore. The experiment report explains how direct observation from the eyes are changed into visual images in the brain. Based on the massive and specialized brain cell array, the fastest and most convenient responses are taken to the four main properties: chromatic sensation, shape and size, depth space, and movement sensation..

There are four manifestations of visual perception in photography creation: composition of a picture, symmetry, balance, and illusion. The purpose of composition of a picture is to enhance the expressive photograph. The symmetrical manifestation means that both sides of the photo by the center line are same or similar characters. Balance manifestation means that the visual image should be balanced features such as size or color, like a physical weight balancing on another object. The illusion is a false feeling to one or more stimuli characteristics, such as size, shape, form, distance, color and movement.

By studying the four main properties of how the brain perceives objects, a conclusion can be made: abstraction is the base of photographic creation and could show up the essence and characteristics of things. If one has a lack of abstraction, then the image will disappear quickly in mind. Therefore, visualization and abstraction are both indispensable in an excellent image.

\section{THE APPLICATION PRINCIPLES AND TECHNIQUES OF EXPRESSION OF VISUAL CONCEPTION IN PHOTOGRAPHY CREATION}

Different visual stimulus can form different integral forms in the perception system. Some forms are balanced that make people feel harmonious. Some forms can cause tension in the perception system, using the counter-acting force to balance the tension and to achieve the stability and simplicity of the image perceived. The process of pursuing balance is in fact the organizing process of perceptual structure. This summarizes many organizational principles that analyzes how the objective is being perceived by the subject.

\section{- Application Principle}

The first principle of expressing the subject of photography is to deal with the relationship between figure and figure-ground. Figure usually refers to the visual elements that can arouse attraction, and figure-ground refers to other parts besides the figure. In photography, the manifestation pattern of highlighting the subject is by using this principle. The attention of figure and figure-ground is in primary and secondary relations. Objects that take less area in the picture, similar objects and integral objects are all easily to be considered as round objects. In addition, the psychological and physical conditions of the viewer also determine which object in the picture to be considered as the figure. Holding the principle of figure and figure-ground relation, we can stress the subject from the figure-ground in the creation of photography and hide the accompaniment body that has no relation with the subject in the figureground.

The principle of similarity refers to: under the condition that objects have the same shape, size, color and strength, visual conception will take these objects as a whole. With the help of the similarity of color, shape, size, texture, location, direction and movement, combine them together by separated elements. These elements will form the rhythm sensation and arouse interests. In folk, ecology and scene photography, many photographers are good at using this principle, forming impressive and interesting pictures.

Complete and closure principle refers to: when an object is in the condition of semi-closure or incomplete picture. The perception system will produce the closure cognition and automatically produce a complete form. This principle in photography creation will construct the tension of the picture. For example, when taking pictures of athletes exchanging the baton, the picture of the next athlete stretching out his hand to take the baton would be better than the picture of holding the baton in his hand. This is the function of this principle.

\section{- Technique of Expression}

Visual synesthesia is the interaction between the visual system and another sensory system such as hearing, feeling, tasting, and smelling. Synesthesia means that the feeling produced by this sensory system is the result of another sensory stimulus. The picture must use an appropriate visual stimulus to stimulate other feelings. When one watches the picture, the eyes are the windows of other feelings. Pictures used in the ads can make you feel thirst, feel the smoothness of an object, experience the good taste of the juicy oranges, smell the fragrance of the perfume, hear the sound of the leaves and so on. A successful photo can arouse different feelings in the viewer. In order to achieve this, he must carefully choose the subject, color, composition, light and so on. Connecting synesthesia with ordinary thinking and language, synesthesia contains the corresponding artistic words, which could stimulate any other or all the feelings.

Personification refers to when non-human or non-living things embody human characteristics. For the photographer that has expressive ability, the most satisfactory form of personification is to set the object in the condition that can reflect the human characteristic. This not only can arouse the excited feeling in both the photographer and viewer. The observant photographers have created all kinds of personified effects by man-made and natural objects, ranging from objects as small as a microorganism to objects as big as a mountain. For example, Edward Weston and Minor White all have successfully captured the beauty from photos of rock structures that resemble parts of human body.

The emotional method refers to the mild and transient feeling in one's mind, which can be aroused or changed by different ways like sight, hearing and other outside stimuli. 
The strongest emotional responses mostly appear in reality. However, feelings aroused by watching a film, a TV program or a photo can be described by language, such as joy, action, hate, uneasiness, boredom, fear, sadness, romance and so on. The visual experience that can be remembered for the longest time and has the strongest influence usually has a relationship with feelings. Light is the basic tool for the photographer to create feelings. Mysterious feelings always stressed by low-key lighting or key light that lower than the location of the vidicon. Diffused reflection light is used in foggy night. In outdoor shooting, in order to wait for the proper light, much time can be wasted. Therefore, artificial light is used to complement the outdoor light or even imitate the outdoor light by the studio light indoors. As for the color photography, the photographer can control the color balance of light at his will by filter, and thus create different feeling effects.

Super realism is an art school, as well as a photography school. It takes the inspiration experience and contingency of Freud as the base, specifically emphasizing the images and unconscious objects related to dreams.

Artists and photographers have taken super realism as a way related to conscious human perception and unconscious feelings of human to the visual sense. The surrealist painter thinks that every object has two sides: common sides, which refers to the aspects that everyone usually view, or delusion and supernatural sides, which refers to the aspects that only a few people can view by the super sight and think in a surreal way. The artistic work must be related with the invisible objects in certain visual form. The techniques used have a wide range, including the analysis of how the thought produced in psychology and the techniques and abilities of the visual artists. The visual effects are very realistic. It is super realistic and yet a dream, just as expressed in the paint of Margaret.

\section{CONCLUSION}

From the physiological function of the eyes, seeing an image does not mean that there is interest in the image or mean that the eyes make high concentration on the image. The camera can mechanically record the outside world, but a human's level to view the image is different from the camera. Although the image effect on the retina is the same with that on the camera, the content of the works is presented in the mind of the audience, and only we perceive the image though the brain's visual thinking. Imagery existence is felt; we truly see this image.

When watching, humans can realize things all from sensory activity. Sensory activity is the simplest way for a human to understand things. In graphic arts, the senses can adjust the effect of the image and extend it to language symbols. In the image language, the image may represent a concept, e.g., the abstract patterns of tobacco pipe may represent the concept of men, and the abstract patterns of high-heeled shoe may represent the concept of women. The different nature of the two concepts is expressed entirely by the abstraction of different patterns. The reason why people make these patterns extend to language symbols, completely depends on the feeling to link one thing with another thing. Feelings make images transfer into a language medium. The image is marked as a concept and considered as a language. Visual language is presented are the characteristics from sensory formation. Although the images can be distributed on the screen, the senses can link the images orderly and the images can be presented through feeling.

Visual arts generally adopt the simple way of structure because only this simplified graphic can present complex relationships. The senses also tend to the simple graphics. Therefore, we mustn't engage in many complex graphics within our creative photography to show the relationship between things. One obtains imagination from the process of feeling and the description of a image. The feeling of things is often the basis for a photographer to perceive and create a work of art. It is also the basis of a person to speak. In modern society, humans generally rely on their senses to perceive, depict and understand the world. Therefore, artistic expression of visual perception has become an important lifestyle in the real world.

Conceptual photography is to pass a certain conception of the creator through camera pictures. Conceptual photography breaks away from the unnecessary, superficial, unconscious entanglement process and uses the means of photography to present the deep analysis for life and the state of humanity. It introduces thought-provoking themes to attract more people. The main purpose of conceptual photography is not to use dogma but to lead a trigger point of many possibilities. This makes viewers comprehend the deeper meaning of a picture. From the viewpoint of reception aesthetic, this is consistent with the needs of aesthetic. If a picture or a group of pictures can't make things more clear, the participation of the audience will be an empty promise and the artistic charm of the photo will be gone. If the audience can read more, it will be a photographic success.

When people treat an image as something they most need, only the visual perception keeps a special relationship between the image and the psychology. Visual art has enduring vitality and a competitive edge in the modern economic society.

\section{REFERENCES}

[1] [UK] John - Berg. The Way of Watching [M], translated by Dai Xingyue. Guilin: Guangxi Normal University Press, 2007.9

[2] [US] James - Elkins. Research on Vision [M], translated by Lei Xin. Nanjing: Jiangsu Fine Arts Publishing House, 2010.1

[3] [Taiwan] Ruan Yizhong. Contemporary Photographers [M]. Beijing: China Photo Press, 2001.1

[4] Ruan Yiqian, Zhong Beili. Psychology [M]. Shanghai: East China Normal University Press .2000.12

[5] [US] Arnheim. Art and Visual Perception [M], translated by Meng Peixin.. Changsha: Hunan Fine Arts Publishing House, 2008.4

[6] Chen Yongguo. Visual Culture Studies Texbook [M]. Beijing: Peking University Press .2009.1

[7] Lin Lu. Special Skill of Photographers [M]. Fuzhou: Fujian Science and Technology Press .2007.4

[8] [UK] Freed. Aesthetics and Photography [M], translated by Wang Shengcai et al. Nanjing: Jiangsu Fine Arts Publishing House, 2008.1 
[9] Lin Yuyuan. Study on Composition of a Picture [M]. Beijing: Higher Education Press .2006.5

[10] [US] Lestes. Visual Communication [M], translated by Cui Wenli. Beijing: Beijing Broadcasting Institute Press, 2003.2

[11] Cao Hui. Aesthetic research on Visual Form [M]. Beijing: People's Publishing House .2009.3

[12] Qian Jiayu. Visual Psychology [M]. Shanghai: Academia Press .2006.1

[13] [UK] James. Elkins. Visual Appreciation [M], translated by Wang Shengcai et al. Guangzhou: Sanlian Bookstore. 2008.1

[14] Caroline .M. Bloomer. Theory of Vision [M], translated by Zhang Gongjin. Beijing: Peking University Press, 1987.

[15] Gregory. Visual Psychology [M], translate by Peng Ranling \& Yang Hao. Beijing: Beijing Normal University Press, 1986. 